\title{
Asymptotics, streamlines, and reservoir modeling: A pathway to production tomography
}

\author{
D. W. VASCO, University of California Berkeley, U.S. \\ AKHIL DATTA-Gupta, Texas A\&M University, College Station, U.S.
}

To in's geophysicists work ever more closely with reservoir engineers and geologists due to the advent of geophysical techniques for reservoir surveillance. Geophysicists have transcended their traditional task of exploration in order to monitor reservoir performance. They are thus called upon to understand the nature of fluid flow within a reservoir and the relationship between reservoir/fluid properties and geophysical observations. It would seem that the physics of fluid flow within a reservoir is, for the most part, unrelated to the phenomena traditionally studied by exploration geophysicists, such as wave propagation within the earth. However, while the physics of fluid flow and elastic-wave propagation are distinct, many shared concepts are extremely useful in reservoir characterization and performance analysis.

In this paper we show a common framework for treating both reservoir engineering and geophysical data, based on asymptotic solutions of the governing differential equations. The asymptotic formulation leads to a practical and efficient methodology, akin to geophysical tomography, for reservoir characterization based on field production data.

Computational challenges in reservoir characterization. Reservoir characterization is an inherently multidisciplinary exercise. Building a high-resolution description of a petroleum reservoir combines the skills of geoscientists and engineers to merge the variety of data collected over its producing life. Such data can be broadly classified as either static or dynamic. Static data consist of time-invariant information such as cores, well-logs, 3-D seismic, and geologic interpretation. Dynamic data are time-varying quantities related to fluid movement within the reservoir and include such information as transient pressure observations, tracer data, multiphase production history, and timelapse seismic measurements. Reconciling static and dynamic data from a reservoir is an important challenge for the industry. It is essential that reservoir models contain the small-scale property variations in well logs and cores while reproducing the large-scale structure and continuity in seismic data. However, a reservoir model derived from these static data usually results in fluid-flow predictions that do not match the production history (dynamic data). Because our ultimate objective is to predict future oil and gas production, it is imperative that such models adequately match all observed flow behavior. For this purpose, a reservoir model constructed from static data typically needs to be conditioned to the dynamic data.

Conditioning reservoir models to dynamic data is equivalent to solving an inverse problem. Such problems are computationally intensive, generally requiring at least an order of magnitude more calculation than needed for forward modeling. By forward modeling we mean reservoir simulation-predicting production given the distribution of reservoir properties. Such predictions can be accomplished using finite difference, finite element, or streamline approaches. Depending on the sophistication

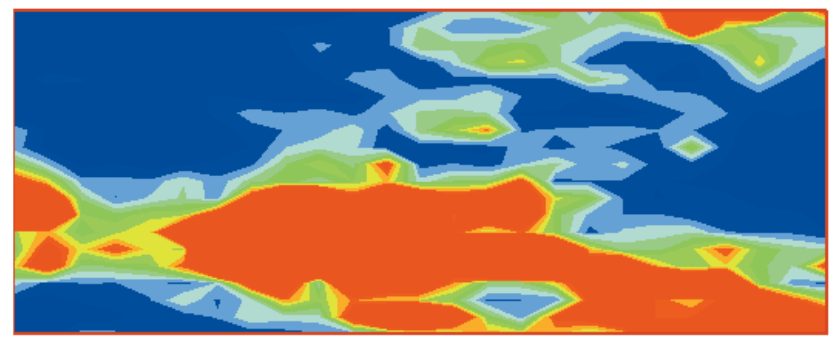

a)
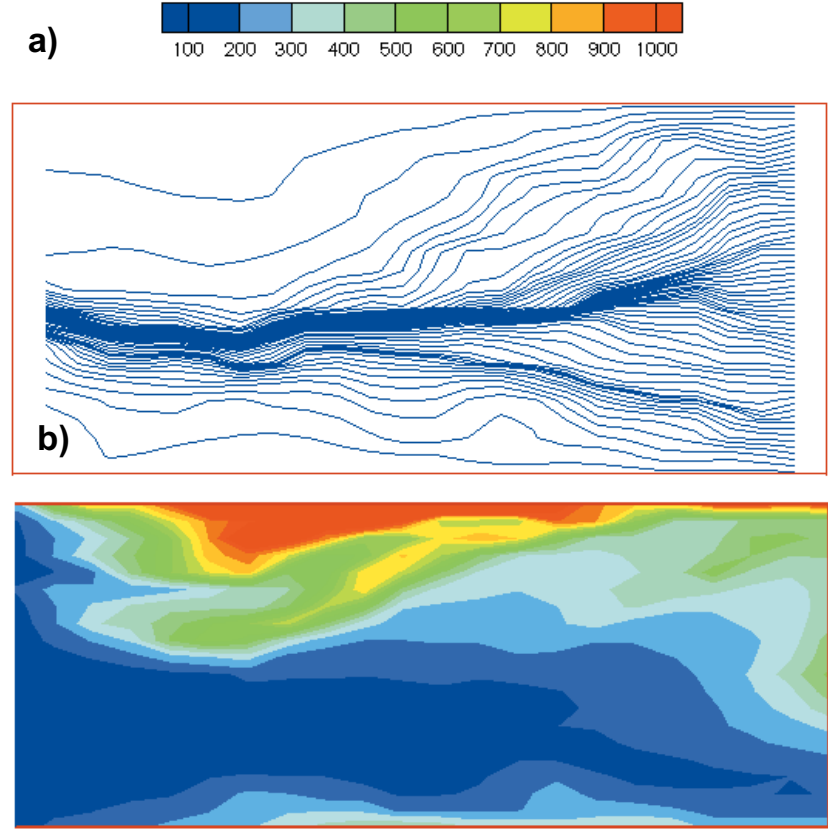

c) $\begin{array}{llllllll}0.00 & 8.57 & 17.14 & 25.71 & 34.29 & 42.86 & 51.43 & 60.00\end{array}$

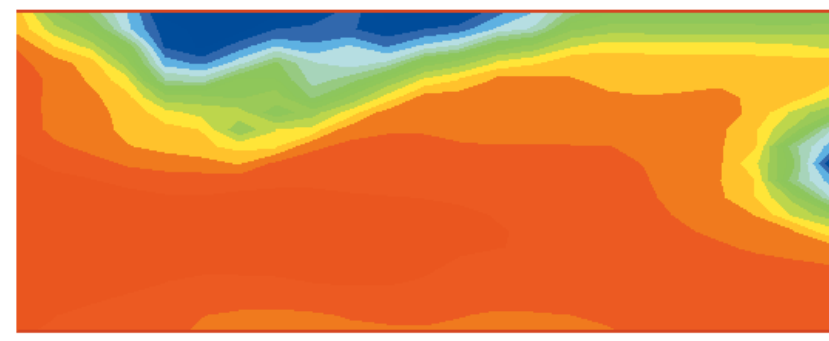

d)

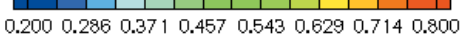

Figure 1. Streamline simulation: a stepwise illustration. Notice the flow channeling along the high-permeability streak as indicated by the time of flight and saturation contours: (a) permeability field (md); (b) streamlines; (c) time of flight (days); and (d) water saturation distribution at 20 days.

and assumptions, the calculations can be substantial. The inverse problem, using production data to estimate reser- 


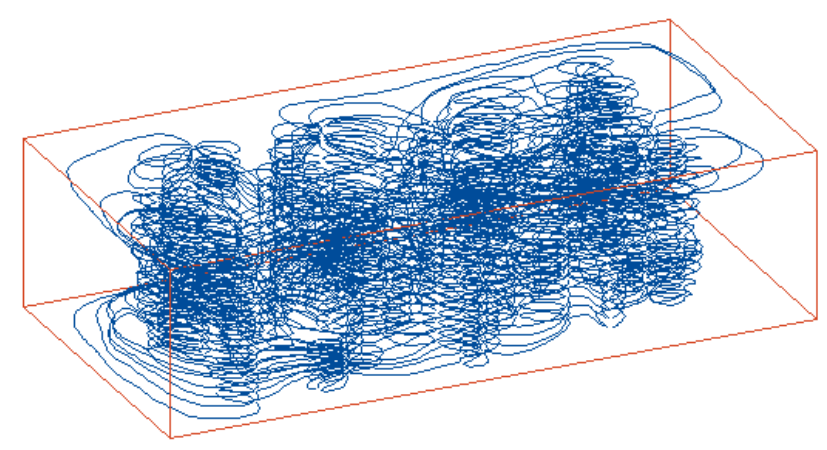

Figure 2. Three-dimensional streamline pattern.

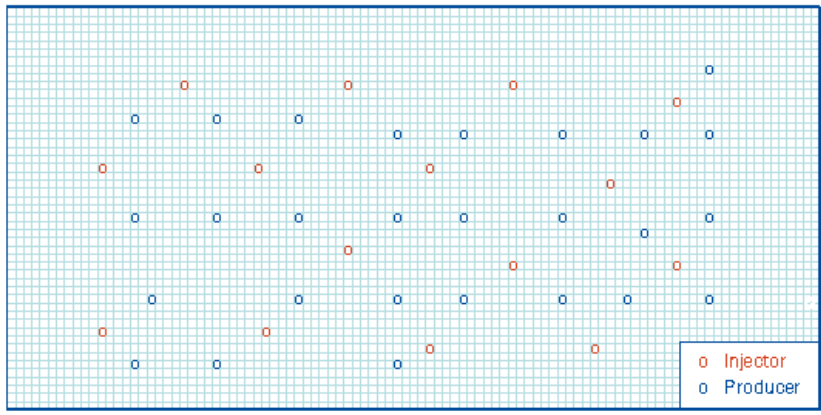

Figure 3. Well configuration for the study area (sections 326 and 327) in NRU.

voir properties, involves repeated reservoir simulation. For example, stochastic approaches, such as simulated annealing, require numerous simulation runs. Similarly, many reservoir simulations are needed to compute sensitivity coefficients required by gradient-based methods. That is, for these iterative inversion methods, we must compute the change in production at a well associated with perturbations of each model parameter. Currently, there are three main approaches for estimating sensitivities: perturbation methods, direct algorithms, and adjoint-state methods. Each has distinct advantages and drawbacks, but all require either extensive computation and/or significant code development. At present all inverse methods for reservoir characterization, stochastic as well as gradientbased, are limited by computational considerations to 100010000 model parameters.

As outlined in this paper, an asymptotic approach leads to an extremely efficient formalism for imaging reservoir flow properties based on field production history. Using this technique we can obtain higher resolution models of reservoir properties of the order of 100000 or more parameters. Asymptotic methods have proven effective in such fields as optics, medical imaging, and seismology. The most important observation is that the sensitivities needed for solving the inverse or imaging problem result from one or a very few forward runs. Thus, such algorithms are orders of magnitude faster than current inversion schemes, which may require hundreds, or even thousands, of reservoir simulations depending on the number of data points and model parameters. Using the asymptotic formalism, large-scale 3-D reservoir imaging problems may be solved in hours rather than days or weeks. Another reason for pursuing the asymptotic formulation is the valuable insight it offers when studying reservoir flow and transport. For example, in applying asymptotic methods to study water flooding, we find that an eikonal equation governs the propagation of the multiphase front. Its analytical form
Initial Match
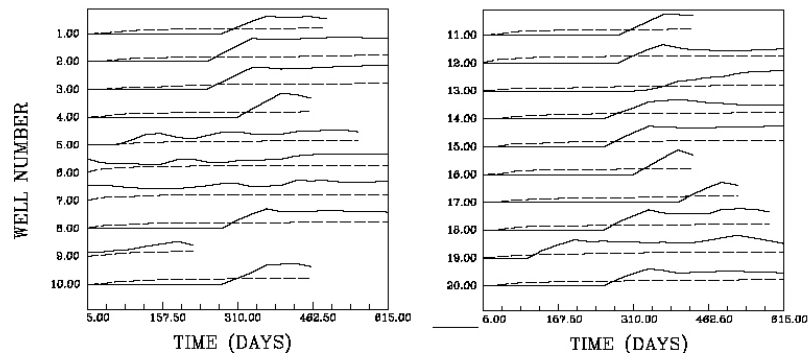

Figure 4. Comparison of observed (solid-line) and predicted (dashed line) water-cuts from NRU. The watercut predictions are based on a layered starting model.

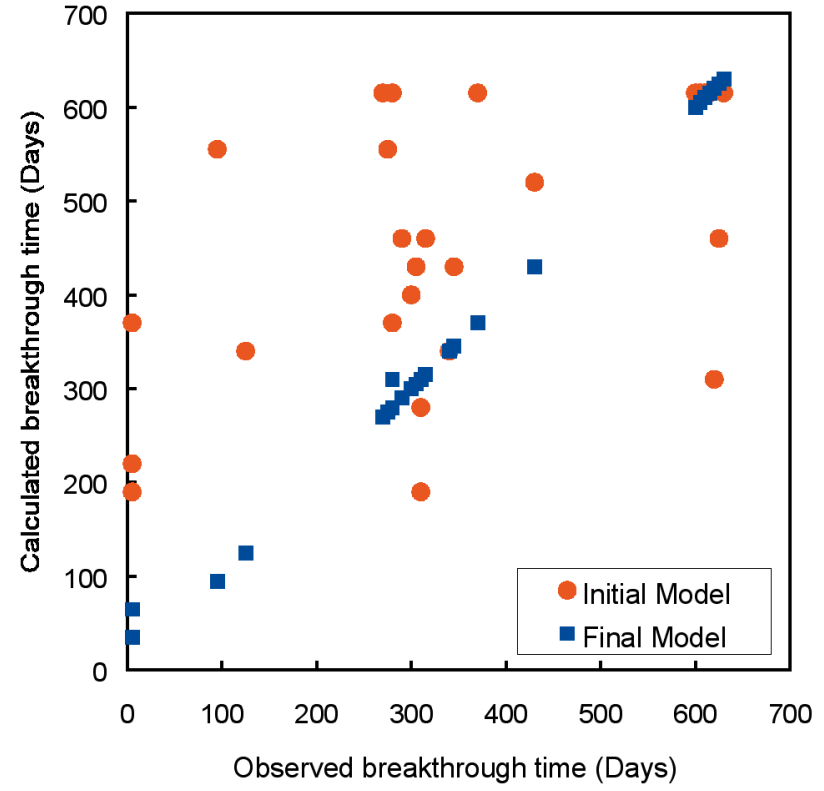

Figure 5. Observed and calculated water breakthrough times at wells before and after inversion. Note that for a perfect match the points would lie along the diagonal (i.e., calculated values would equal observed values).

clearly indicates that particular combination of flow properties (saturation, pressure, porosity, permeability, relative permeability) influencing the arrival time of the water front arrival time. Such insight is essential for understanding the trade-offs and uncertainties inherent in using production data to image reservoir flow properties.

Asymptotic solutions for flow and transport. The asymptotic approach is based on the assumption that two or more spatial-temporal scales are at play in the propagation of a wave or a fluid interface. For example, when water is injected into a producing horizon, it generally forms a self-sharpening front. The front separates the unswept reservoir rock from the water-flooded portion of the reservoir. Length and time scales associated with the transition from oil-saturated to water-flooded rock are typically much less than length and time scales over which the background oil and water saturations change. We can construct an asymptotic solution to the equations for multiphase flow in terms of the ratio of these length scales, as we might do for a high-frequency seismic or electromagnetic wave. For example, we can represent the saturation 
Final Match
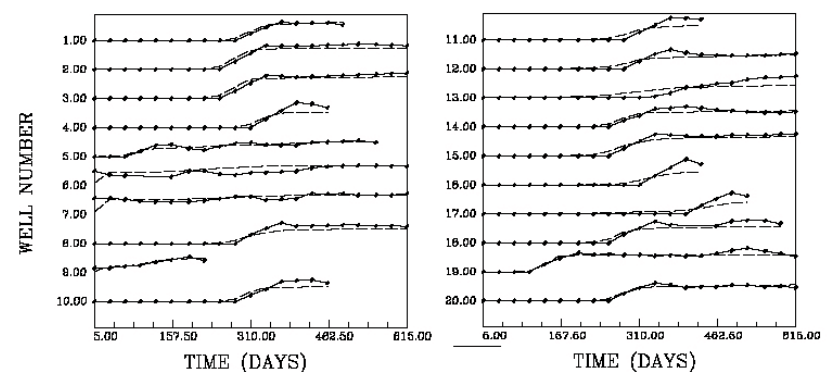

Figure 6. Observed (solid-line) and predicted (dashedline) water-cut based on final estimates of porosity and permeability.

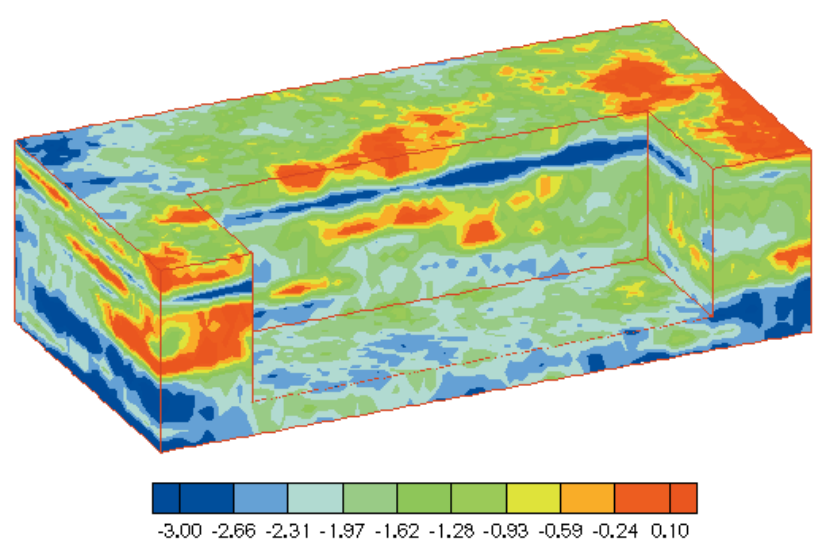

Figure 7. Permeability field after water breakthrough time match (logarithmic scale).

associated with a two-phase front, in the form of a sum of the background saturation variation and an infinite sum. (See equation 1 of the Appendix for a complete description.)

In most cases we are only interested in the first one or two terms of the summation. For example, the first term in the expansion is related to the phase, or traveltime, of the propagating front. The spatial variation of the phase is governed by a scalar eikonal equation, in the same fashion as a seismic wave. In solving the eikonal equation, we derive a set of ray equations that define trajectories between producing and injecting wells. For two-phase flow, the trajectories describe the path taken by the two-phase front in propagating from the injector to the producer. The traveltime of the two-phase front is primarily influenced by variations in properties along this path. As we shall soon see, this fact is of great consequence in developing an efficient algorithm for solving the inverse problem. By reformulating the equations in traveltime coordinates, we can derive an ordinary differential equation (the transport equation) describing the evolution of the amplitude of the two-phase front. Thus, we can compute the saturation evolution by a series of one-dimensional calculations along the trajectories. Again, the situation is analogous to seismic ray methods.

Asymptotic solutions and efficient reservoir characterization. Using the asymptotic formalism, we can develop a very cost-effective technique for history matching reservoir production data. In doing so we take advantage of knowledge gleaned from fields such as medical imaging and geophysical tomography. In particular, we can partition the production history matching problem into two subproblems: an arrival-time inversion and an amplitude inversion. The benefits are identical to waveform inversion in seismology: Amplitude matching is strongly nonlinear and its success very much depends on an adequate starting model. Furthermore, seismic amplitudes are significantly influenced by properties at the source and receiver locations.

Similar issues arise in the inversion of dynamic data. For example, transient pressure amplitude measurements can be strongly effected by well-bore storage. Thus, we start by matching the arrival times of the dynamic data. For example, tracer or water-cut breakthrough times or the time associated with the peak slope of transient pressure observations. In inverting the arrival times, we make use of the analytic sensitivities provided by the asymptotic formalism. These quantities are similar to those used in seismic tomography: the path length in each block of the reservoir model divided by the square of the velocity. The velocity follows from the particular form of the eikonal equation and will depend on the type of dynamic data considered (transient pressure, tracer, water-cut). We shall illustrate this by several examples.

Upon successful arrival-time matching, we turn to the more difficult amplitude inversion. However, we may use the reservoir model produced by the arrival-time match as our starting point. Again, we can make use of analytic sensitivities provided by the asymptotic solution. It turns out that, to the lowest order approximation, the amplitude sensitivities are in a form much like those for traveltimes. Thus, having defined the trajectories, we can compute the amplitude sensitivities by evaluating simple analytical expressions along these paths.

Let us briefly summarize the steps in our inversion strategy. First, we conduct a reservoir simulation to compute the spatial and temporal variation in reservoir pressures, saturations, etc. Then, we solve the ray equations numerically to define the trajectories. At this stage we have all quantities necessary to conduct both traveltime and amplitude inversions. We then solve the linearized system of equations for the model parameter perturbations and update the reservoir model. Because we solve the inverse problem iteratively, we repeat this procedure until convergence (until the data are matched).

Coupling asymptotic solutions and streamline simulation. Asymptotic approaches in seismology have relied on defining raypaths or trajectories associated with wavefronts. For fluid-flow modeling, such trajectories can often be identified with streamlines, instantaneous flowpaths locally tangential to the fluid velocity. Streamlines are perpendicular to a fluid front in a manner analogous to seismic rays. Thus, it makes sense to couple the asymptotic approach to streamline simulation. The basic steps involved in streamline simulation are: (1) tracing streamlines based upon a computed pressure field, typically derived numerically using finite difference or finite element methods; (2) computing particle traveltime or time of flight along streamlines; (3) decoupling the transport equations (concentration and saturation equations) using a coordinate transformation from physical space to the time of flight coordinates following flow directions; (4) solving the resulting one-dimensional transport equations along the streamlines; and (5) updating the streamlines as necessary, to account for mobility effects or changing field conditions (Figure 1). 


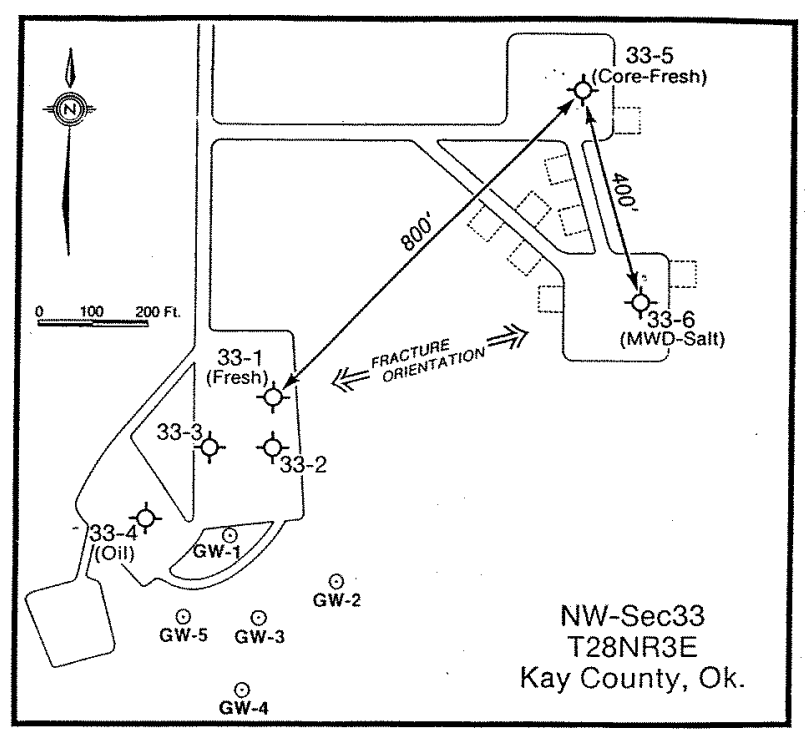

Figure 8. Conoco Borehole Test Facility.

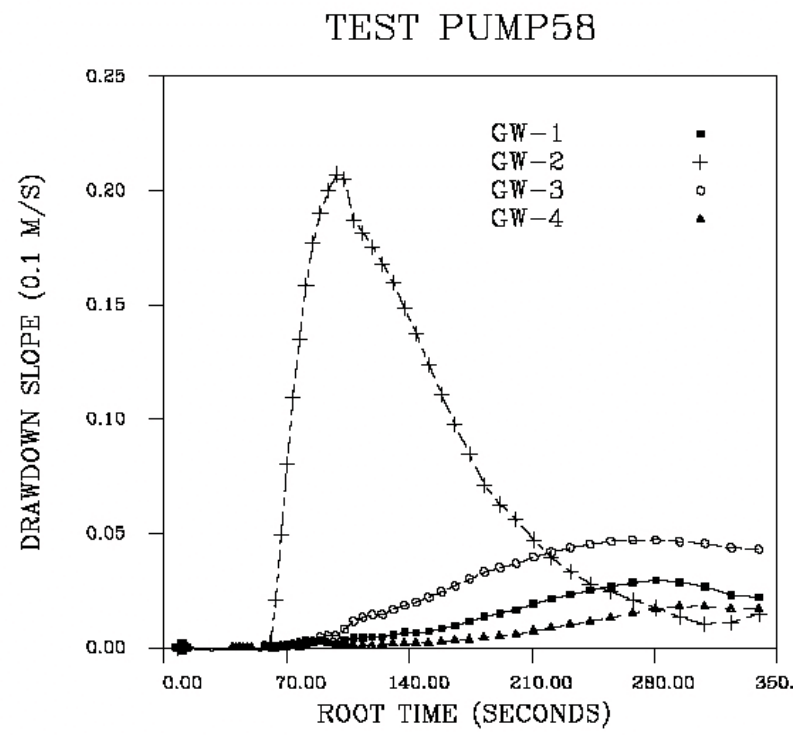

Figure 9. Pressure drawdown derivatives at the observation wells during interference test Pump58. (a) Initial match. (b) Final match. (c) Final permeability distribution.

Notice the grouping of streamlines along the high-permeability streak and the flow channeling as indicated by the time of flight and saturation contours. In particular, the time of flight coordinate offers a natural and quantitative way of flow visualization that provides a mechanism for dynamic reservoir characterization. The computational advantage of the streamline approach can be attributed to the fact that streamlines need to be updated only infrequently and the transport equations along streamlines are decoupled from the underlying grid, thus allowing for faster solution. Furthermore, the self-similarity of the solution along streamlines may allow us to compute the solution only once and map it to the time of interest. The disadvantages of streamline models are the difficulties in incorporating complex physical processes and crossstreamline mechanisms. In three-dimensions, the configuration of streamlines can become quite complicated for realistic production scenarios. For example, in Figure 2 we display the streamlines associated with production in the
North Robertson Unit described below.

Multiphase flow and the interpretation of water-cut measurements. We now illustrate the utility of the asymptotic approach by considering water-cut data from a producing oil field. For the majority of oil fields, water-cut observations are the primary source of information related to multiphase flow within the reservoir. Water-cut simply refers to the fraction of water in the fluid produced from a well. Water-cut is routinely measured on a monthly basis in most fields. In our interpretation of these data, we must contend with the equations describing multiphase flow, a set of nonlinear partial differential equations. We may derive asymptotic solutions to these equations under the assumption that the multiphase fronts are sharp in comparison to the background heterogeneity. If this assumption is valid, the time needed for a front of injected water to travel from the injector to a producer can be expressed simply as an integral along the path followed by the water, as determined by the pressure gradient and relative mobility-in turn controlled by the relative permeabilities and viscosities of the oil-water system. (See equation 2 in the Appendix). We observe that the arrival time of the twophase front is influenced by the ratio of porosity to permeability within the reservoir. The asymptotic methodology also allows us to derive analytic expressions for amplitude sensitivities, relating perturbations in reservoir porosity and permeability to variations in water-cut amplitudes as a function of time. Given the complete production history of a reservoir, we may piece together a picture of the large-scale interwell heterogeneity.

Our initial application of the asymptotic approach was to water-cut measurements from the North Robertson Unit (NRU), a heterogeneous carbonate reservoir within the Permian Basin of west Texas. As with the majority of such reservoirs, there are a variety of production problems such as lack of continuity, low water-flood sweep efficiency, early water breakthrough, and water channeling. The nonreservoir rock types are relatively impermeable and form vertical barriers contributing to reservoir heterogeneity and compartmentalization. Identification of the location and distribution of these barriers is critical to the success of secondary and tertiary recovery. The presence of fractures contributes to the heterogeneity, further complicating the production response. Finally, available reservoir performance data are limited, particularly for older wells. Two sections of the NRU, 326 and 327, were selected for detailed study (Figure 3 ). The reservoir model consists of a $100 \times 50 \times 10$ grid of cells. We allowed both permeability and porosity to vary in each cell, resulting in a total of 100 000 model parameters. Altogether 42 wells (27 producers and 15 injectors) from the study area were used to characterize heterogeneity based on the water-cut response from the producing wells. An initial model was constructed using geostatistical methods, based on well-log data from 30 boreholes. Figure 4 compares the predicted water breakthrough histories at the producing wells, based on the initial model, with the observed production histories. The arrival-time matching was completed in 30 iterations. In Figure 5 we compare observed breakthrough times with predictions based on both our initial and final (arrival-time inversion) models. Clearly, the breakthrough times predicted by the inversion result are now in complete agreement with the field data. It took less than two hours of computation on a PC to match these arrival times. An additional evening of computation was required to match the details of the water-cut amplitudes themselves (Figure 
a)

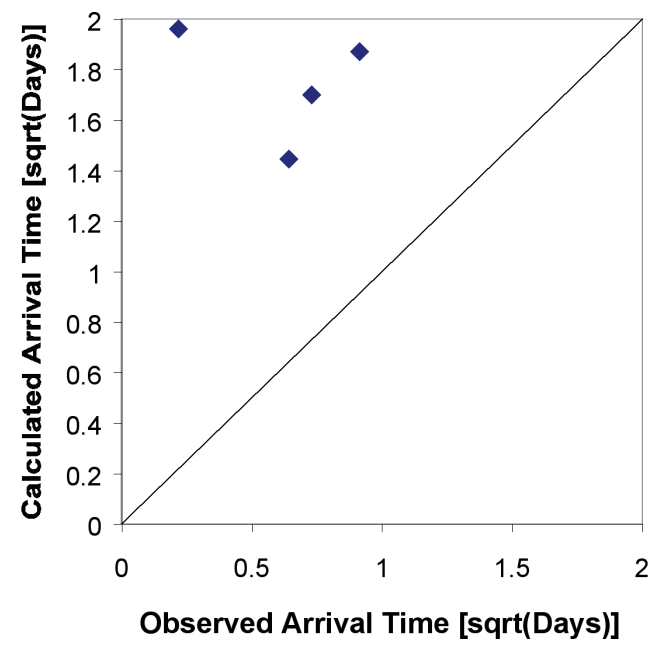

b)

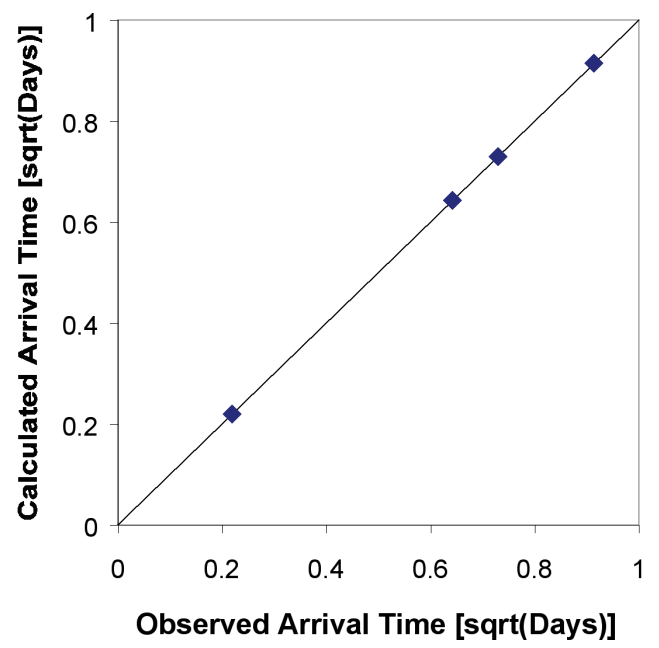

c)

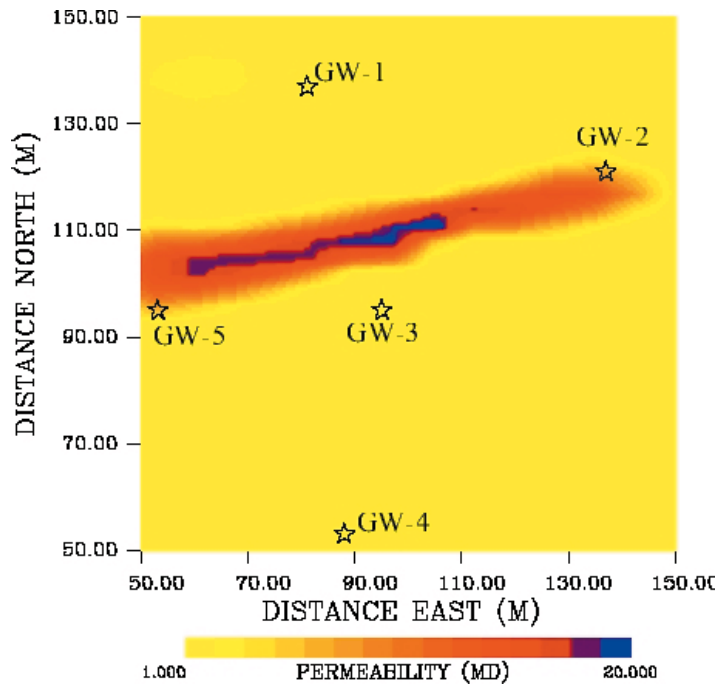

Figure 10. Fracture zone characterization based on an inversion of the Conoco transient pressure data: (a) initial match; (b) final match; and (c) final permeability distribution.

6). We found that the amplitude inversion primarily changed the magnitude of the porosity and permeability anomalies, but not the overall pattern. Figure 7 shows the permeability variations, after incorporating the water breakthrough responses. The permeability field derived from this inversion is consistent with an earlier study. This investigation, based on a conventional decline curve analysis, found that section 326 had the best production characteristics overall. This agrees with the generally higher permeability we infer in this section.

Pressure inversion and diffusive tomography. Asymptotic methods are also useful in the analysis of diffusive phenomena, such as transient pressure response. A transient pressure signal is generated when a well comes on line, or is shutdown. This signal, typically a step-like change in flow rate and pressure, varies more rapidly than does the background pressure field. We can use this difference in scale to construct an asymptotic series solution. The trajectory, described by the ray equations, is the path taken by the first-arriving pressure transient (equation 3 in the Appendix). Traveltime to each point along the path is determined by the ratio of the reservoir storage to the hydraulic conductivity. Knowing this relationship, we can perform transient pressure traveltime tomography. That is, we can utilize concepts such as back-projection and sparse matrix methods to construct an efficient algorithm for mapping arrival time anomalies into lateral variations of the ratio of reservoir storage to hydraulic conductivity.

We applied the tomographic pressure inversion algorithm to measurements from two interference tests conducted at the Conoco Borehole Test Facility in Kay County, Oklahoma. Wells GW-1 through GW-5, which form a skewed 5-spot (Figure 8), penetrate the Fort Riley Formation, a fractured limestone. The formation is part of the Lower Permian Chase Group that consists primarily of limestones and shales. Average permeability of the Fort Riley itself is about $1.2 \mathrm{mD}$. The interference tests involve fluid withdrawal, at a constant rate of 2.3 liters/minute. In the first test, water is extracted from well GW-5. In the second test GW-2 is the producing well. During pumping, the pressure responses are recorded in the surrounding wells. One advantage of the asymptotic approach is that it enables us to define "pressure fronts" and "arrival times" associated with these pressure transients. In order to define an arrival time, we work with the time derivative of pressure rather than the pressure itself. The derivative is used because the sources are step functions, rather than sharp pulses. The pressure time differences for test Pump58 (pumping from GW-5) are shown in Figure 9. Note the great variation in the arrival time of the peaks, with the nearby well GW-3 showing much later arrival time than the more distant GW-2. Our starting permeability model consists of a homogeneous layer. Traveltime predictions, based on this uniform initial model, are much larger than the observed traveltimes (Figure 10a). After five iterations of our tomographic algorithm, we have an excellent fit to the observed traveltimes (Figure 10b). Using the arrival-time inversion result as a starting point, we then fit the amplitudes of the pressure responses. The inferred permeability field is a dominant fracture in an east-northeast direction to the north of well GW-3 (Figure 10c). Independent geophysical experiments verified both the existence and location of the fracture with respect to well GW-3. Seismic amplitude attenuation and single-well imaging indicated a fracture or fracture zone to the north of GW-3. Final confirmation of the fracture came from a slant well, slightly to the west of the central well GW-3. Core from the slant well verified the location of the fracture zone (Figure 11).

Tracers and partitioning tracers. As we have seen, the asymptotic methodology is applicable to routinely gathered production data, such as water-cut observations. The 


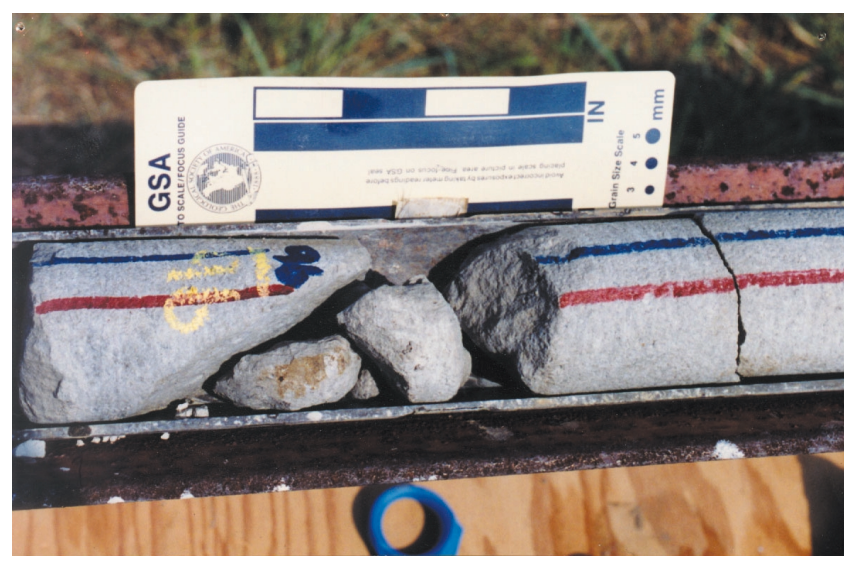

Figure 11. Slant well core containing fracture zone.

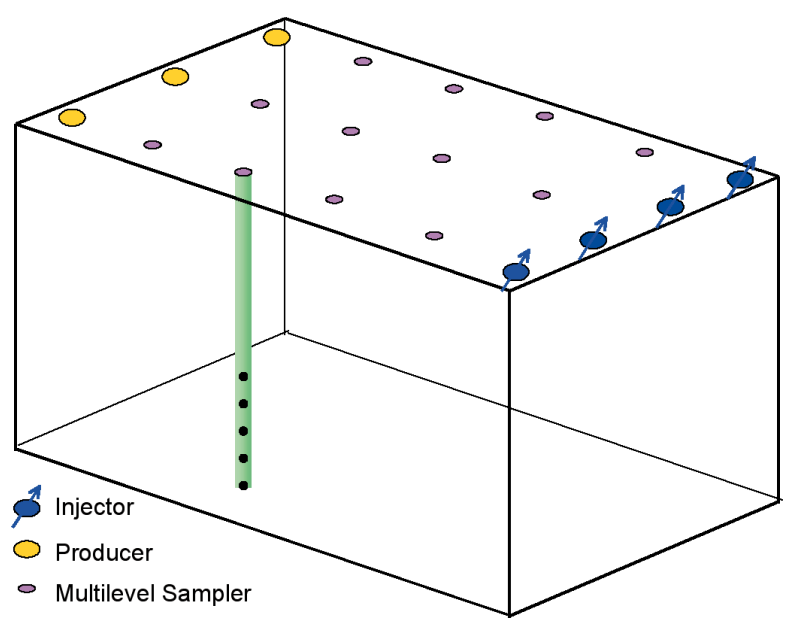

Figure 12. Hill Airforce Base test cell diagram.

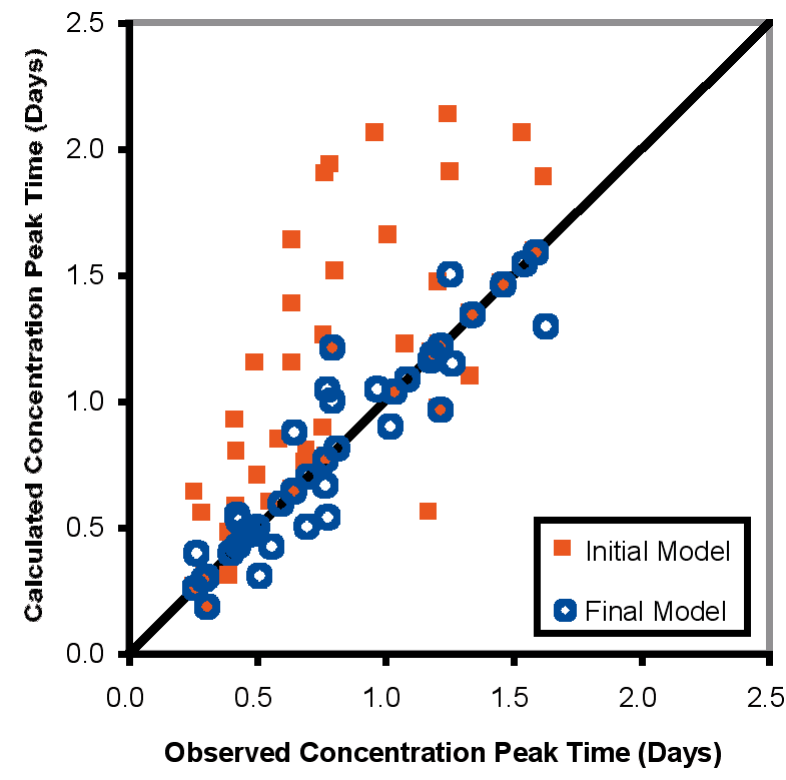

Figure 13. Observed and calculated concentration peak arrival times at all sampling locations before and after inversion for NAPL saturation.

technique is also extremely useful in the analysis of measurements from more specialized experiments, such as tracer tests and partitioning tracer tests. In these tests, a small slug of an observable tracer is added to the injected fluid. Produced fluids are then sampled at particular time intervals and analyzed for the concentration of the injected tracer. The arrival time and concentration history of the tracer may be used to infer flow properties between wells using an expression similar to that used previously for the water-cut data (equation 4 in the Appendix).

Partitioning tracer experiments involve injection of two tracers: one is "conservative" and does not react with in situ pore fluids; the other tracer is "partitioning" that is, partly soluble in the resident pore fluids and is, thus, characterized by a delayed arrival time. The utility of the partitioning tracer test rests on the fact that the temporal or "chromatographic" separation between the two tracer responses at the producing wells is a measure of the average saturation of the resident pore fluid, integrated along the flow path (equation 5 in the Appendix). Given adequate data, we can infer the large-scale saturation distribution in the interwell region. The inversion proceeds in two steps. First, conservative tracer data (and any other available information) are used to constrain the large-scale permeability variation between the wells. Fixing this, we use the partitioning tracer responses to infer saturation variations in the subsurface. If necessary, we iterate between matching the conservative and partitioning tracer measurements, consequently updating the permeability and saturation variations.

We have applied our asymptotic methodology to both conservative and partitioning tracer data. Here, we focus on recent results from a partitioning tracer experiment conducted at Hill Air Force Base in Utah. This experiment was associated with an environmental application, identifying the distribution of a nonaqueous-phase-liquid (NAPL) in the subsurface. In this experiment, both conservative and partitioning tracers were introduced into four injection wells and subsequent concentration histories recorded in 12 multilevel sampling wells and three extraction wells (Figure 12). Tracer concentrations are sampled at five depth intervals in each borehole, giving 3-D coverage. In total there were 51 tracer responses in the sampling and extraction wells. The initial fit to the reactive tracer is shown in Figure 13. Using equation 5 in the Appendix, we may infer both the ratio of porosity to permeability and the saturation distribution within the aquifer. The complete arrival-time inversion for saturation took only 94 s on a Pentium III PC. The resulting NAPL saturation distribution is portrayed as a cutaway view in Figure 14. We infer an average NAPL saturation of about $6 \%$, with higher saturation toward the lower part of the test cell. The spatial distribution of NAPL appears consistent with soil core analysis, indicating higher NAPL saturation clustered toward the lower portion of the cell. The final fits to the observed arrival times are shown in Figure 13. The improvement in fit is significant, and the predicted traveltimes match the observed values quite well.

Model assessment. In contrast to geophysical data sets, production data are often limited in both lateral and vertical distribution. Obviously, the measurements are restricted to wells. In some situations the produced fluids from several wells are commingled, further reducing the resolution provided by the data. Water-cut measurements frequently average over the entire reservoir interval or over many producing zones. This severely limits vertical resolution of reservoir flow properties. By their nature, production data average along the flow paths. As mentioned, it is possible to combine geophysical and production data to extend the "sampling" to interwell regions, but such 


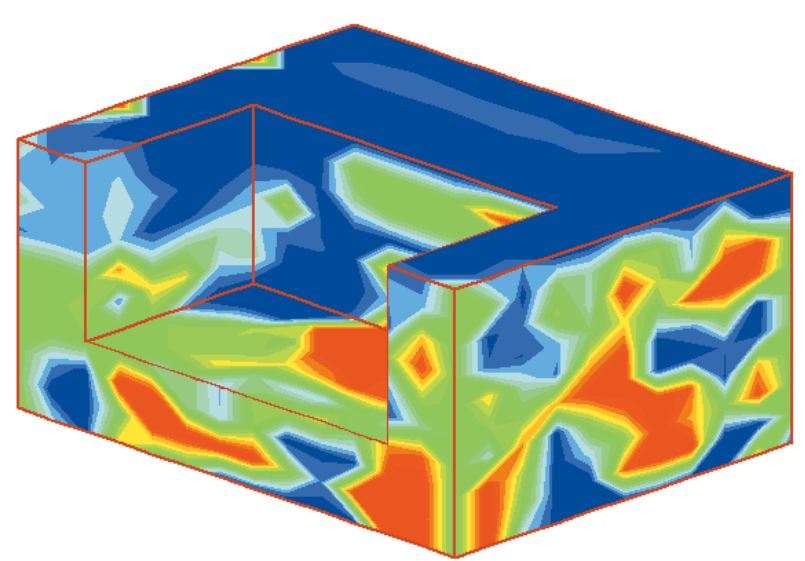

\begin{tabular}{|l|l|l|l|l|l|l|l|l|l|l|l|l|l|l|}
\hline & & & & & & & & & & & & & & \\
0.000 & 0.009 & 0.017 & 0.026 & 0.034 & 0.043 & 0.051 & 0.060 & 0.069 & 0.077 & 0.086 & 0.094 & 0.103 & 0.111 & 0.120
\end{tabular}

Figure 14. NAPL saturation distribution estimated from partitioning tracer data for the Hill case.

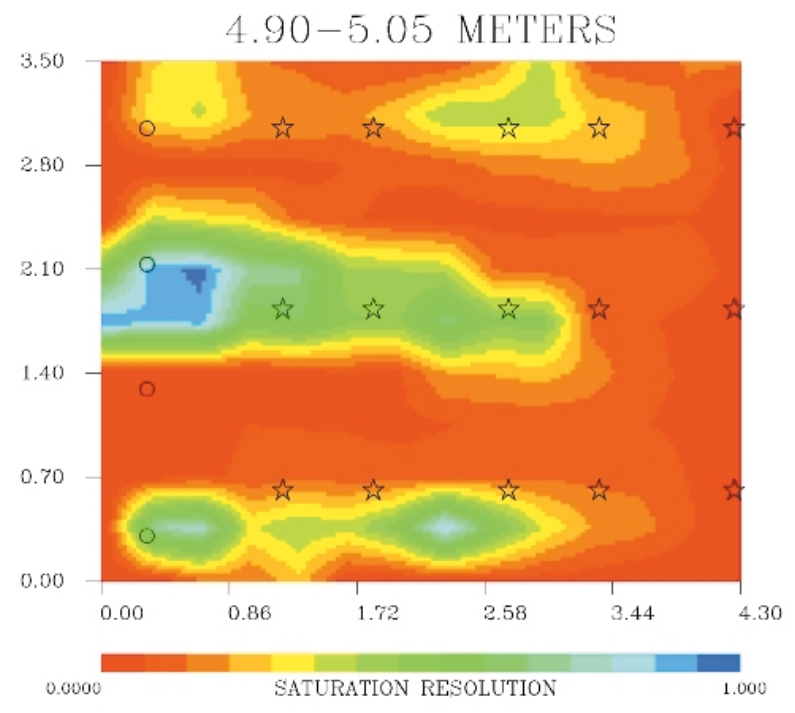

Figure 15. Resolution associated with saturation estimates for the third layer of the Hill model.

work is still relatively uncommon. These factors highlight the importance of conducting some form of model assessment in conjunction with estimates of reservoir flow properties. Fortunately, the asymptotic approach lends itself to efficient computation of model parameter resolution and uncertainty. Specifically, the sensitivities are computed along the trajectories, much like raypaths. The intersection of these paths with the cells of the reservoir model result in quite sparse sensitivity matrices, much like those of seismic traveltime tomography. Thus, one may use sparse matrix methods to construct resolution and covariance estimates. Sparse matrix methods allow us to reduce the storage requirements and the corresponding number of floating point operations necessary to conduct a model assessment.

As an example, consider the resolution associated with our saturation estimates obtained for the Hill Air Force Base tracer tests. Using sparse matrix techniques, we may compute resolution estimates without conducting a matrix inversion or a formal singular-value-decomposition (SVD). Rather, there are iterative algorithms that enable us to construct a partial SVD. In the case of the Hill experiments, Figure 15 shows the resolution of saturation estimates based on the partitioning tracer data. Higher resolution is observed along those flow paths encountering multilevel samplers, where sensitivities are larger. At locations where the resolution is close to zero, between the rows of samplers, it is not possible to estimate the saturation variations reliably.

Conclusions. Reservoir models continue to grow in size and sophistication and multimillion-block models are now commonplace. Supporting geophysical observations, such as time-lapse 3-D seismic data, provide large volumes of information that can constrain fluid-front propagation in the reservoir. To effectively integrate diverse sources of information into high-resolution reservoir descriptions, we need flexible and efficient schemes for reservoir modeling and characterization. Asymptotic solutions for flow and transport offer advantages over conventional history matching and data integration. First, there is the added flexibility provided by explicit expressions for traveltimes and amplitudes. Thus, we can formulate two-step inversions in which we first match arrival times and then match the history data themselves. The arrival-time expressions can be very helpful in integrating time-lapse seismic data. That is, we can directly relate time-lapse images of a multiphase front to reservoir porosity and permeability. By implementing the asymptotic methodology in a streamline simulator, we obtain the efficiency necessary for handling large reservoir models. In particular, trajectory methods rely on one-dimensional paths through the reservoir model. Therefore, we only require storage and computations for those cells intersected by a particular trajectory. For this reason, the methods scale quite well with the size of the reservoir model. Though our initial applications involved tens to hundreds of thousands of model parameters, million-cell models are certainly within our reach. The semianalytic sensitivities provided by the asymptotic formulation mean that we can conduct one inversion step based on a single reservoir simulation. This is a very significant step toward making dynamic data integration a routine part of reservoir characterization.

Suggested reading. "Streamline simulation: A technology update" by Datta-Gupta (SPE Distinguished Author Series, Journal of Petroleum Technology, 2000). "Streamlines, ray tracing and production tomography: Generalization to compressible flow" by Datta-Gupta et al. (Petroleum Geoscience, 2001). "Resolution and uncertainty in hydrologic characterization" by Vasco et al. (Water Resources Research, 1997). "Asymptotic solutions for solute transport: A formalism for tracer tomography" by Vasco and Datta-Gupta (Water Resources Research, 1999). "Estimation of reservoir properties using transient pressure data: An asymptotic approach" by Vasco et al. (Water Resources Research, 2000). "Asymptotics, saturation fronts, and high resolution reservoir characterization" by Vasco and Datta-Gupta (Transport in Porous Media, 2001). E

Acknowledgments: This work is based on a collaborative research project of the Lawrence Berkeley Laboratory, Texas A\&M University, and multiple industry partners. This work is supported by the Assistant Secretary for Fossil Energy, Office of Natural Gas and Petroleum Technology, through the Natural Gas and Oil Technology Partnership, under U.S. Department of Energy Contract DE-AC03-76SF00098. The authors thank Bob Lemmon from the National Petroleum Technology Office for his continued support and encouragement.

Corresponding author: 


\section{Appendix: Equations and Formal Presentation}

Equation 1: We can represent the saturation, $S(\mathrm{x}, t)$, associated with a two-phase front, in the form of an infinite sum

$$
S(x, t)=S_{0}(x, t)+\sum_{n=1}^{\infty} \varepsilon^{n} S_{n}(x, t)
$$

where $S_{0}(\mathrm{x}, t)$ is the background saturation variation and $S_{n}(\mathrm{x}, t)$ are functions which represent successively more rapidly varying components of the saturation distribution.

Equation 2: The time needed for a front of injected water to travel from the injector to a producer can be expressed simply as an integral or summation along the trajectory $\Sigma$

$$
T=\int_{\Sigma} \frac{1}{\lambda|\nabla P|} \frac{n(r)}{k(r)} d r
$$

where $n(r)$ is the porosity along the trajectory and $k(r)$ is the permeability. The pressure gradient is denoted by $\nabla \mathrm{P}$ and ( represents the total relative mobility given by the relative permeabilities and viscosities of the oil-water system.

Equation 3: The square root of the travel time from the source or injection well to an observation well, $T(x)$, is given by a line integral along this trajectory,

$$
\sqrt{T(x)}=\int_{\Sigma} \sqrt{\Delta(r)} d r .
$$

In this expression $\Sigma$ is the trajectory from the source to the observation point and $\Delta(r)$ is the ratio of reservoir storage $\Theta$ to the hydraulic conductivity $k$,

$$
\Delta(r)=\frac{\Theta(r)}{k(r)}
$$

which is a function of distance $r$ along the flow path.

Equation 4: The arrival time and concentration history of the tracer may be used to infer flow properties between wells. In particular, the arrival time of the tracer is given by an integral along the flow path $\Sigma$,

$$
T_{c}=\int_{\Sigma} \frac{1}{|\nabla P|} \frac{n(r)}{k(r)} d r .
$$

This expression for traveltime is similar to the relationship for water-cut data. For single-phase tracer flow we can neglect relative permeability effects, as contained in the term $\lambda$.

Equation 5: The specific equation for the traveltime of the partitioning tracer is

$$
T_{c}=\int_{\Sigma} \frac{1}{|\nabla P|} \frac{n(r)}{k(r)}\left[S_{w}+K_{O} S_{O}\right] d r
$$

where $S_{w}$ denotes the water saturation, $S_{0}$ represents the oil saturation, and $K_{0}$ is the known partitioning coefficient that defines the relative solubility of the tracer in the injected and resident fluid. 